\title{
Renal involvement in children and adolescents with inflammatory bowel disease
}

Hea Min Jang, MD', Hee Sun Baek, MD', Jung-Eun Kim, MD', Ju Young Kim, MD', Yeon Hee Lee, MD², Hee Yeon Cho, MD², Yon Ho Choe, $\mathrm{MD}^{2}$, Ben Kang, MD', Byung-Ho Choe, MD', Bong Seok Choi, MD', Min Hyun Cho, MD'1

${ }^{1}$ Department of Pediatrics, School of Medicine, Kyungpook National University, Daegu, ${ }^{2}$ Department of Pediatrics, Samsung Medical Center, Sungkyunkwan University School of Medicine, Seoul, Korea

Purpose: The incidence of inflammatory bowel disease (IBD) is rapidly increasing, and several reports have described the renal complications of IBD. We sought to evaluate the clinical manifestations of renal complications in children with IBD in order to enable early detection and prompt treatment of the complications.

Methods: We retrospectively reviewed the medical records of 456 children and adolescents aged $<20$ years who had been diagnosed with IBD since 2000 . We analyzed patient age, sex, medication use, IBD disease activity, and clinical manifestations of renal symptoms.

Results: Our study comprising 456 children with IBD included 299 boys (65.6\%) and 157 girls (34.4\%). The study included 346 children with Crohn disease and 110 children with ulcerative colitis. The incidence of kidney-related symptoms was $14.7 \%$, which was significantly higher than that in normal children. We observed 26 children (38.8\%) with isolated hematuria, 30 children (44.8\%) with isolated proteinuria, and 11 children (16.4\%) with hematuria and concomitant proteinuria. A renal biopsy was performed in 7 children. Histopathological examination revealed immunoglobulin A nephropathy in 5 children (71.4\%). All children presented with mild disease and well-controlled disease activity of IBD.

Conclusion: Children with IBD are more likely to show kidney-related symptoms than healthy children and adolescents are. Therefore, regular screening of urine and evaluation of renal function in such children are necessary for early detection of renal complications.

Key words: Inflammatory bowel disease, Kidney, Complication, Child, Adolescent

\section{Introduction}

Inflammatory bowel disease (IBD) is a chronic inflammation of the gastrointestinal tract that presents with recurring bouts of inflammation and remission. The 2 most common types of IBD are Crohn disease (CD) and ulcerative colitis (UC). ${ }^{1,2)}$ Pediatric IBD, which was previously rare in Koreans, has shown a rapid rise in incidence after the 1990s, with a pronounced increase over the last 5 years. The age of onset of IBD is 15-25 years, and CD is more common in children than in adults. ${ }^{1)}$ Extraintestinal manifestations are varied from 25\% to 40\% in both adults and children with IBD, but kidney and lower genitourinary involvement has been reported in 4\%$23 \%$ of adult patients with IBD and 1\%-2\% of children with $\mathrm{BDD}^{2)}$ Urinary calculi, fistulas, and renal tubular damage are known to occur, and although renal parenchymal disease is relatively rare, cases showing glomerulonephritis (GN), minimal-change disease (MCD), secondary (AAtype) amyloidosis, and interstitial nephritis are being commonly reported. Based on references described in the literature, immunoglobulin A (IgA) nephropathy accounts for the largest percentage of disease at 24\%, followed by interstitial nephritis (19\%), acute tubular injury (8\%),
Corresponding author: Min Hyun Cho, MD Department of Pediatrics, Kyungpook National University Hospital, 130 Dongdeok-ro, Jung-gu, Daegu 41944, Korea Tel: +82-53-200-5719

Fax: +82-53-425-6683

E-mail:chomh@knu.ac.kr https://orcid.org/0000-0002-8695-5558

Received: 28 March, 2018

Revised: 20 June, 2018

Accepted: 25 June, 2018

Copyright @ 2018 by The Korean Pediatric Society

This is an open-access article distributed under the terms of the Creative Commons Attribution NonCommercial License (http://creativecommons.org/ licenses/by-nc/4.0/n which permits unrestricted noncommercial use, distribution, and reproduction in any medium, provided the original work is properly cited. 
proliferative GN (7\%), and MCD (5\%).

We evaluated the clinical manifestations of renal complications in children and adolescents with IBD in 2 tertiary centers in Korea to enable early detection and prompt treatment of renal complications.

\section{Materials and methods}

We retrospectively reviewed the medical records of 456 children and adolescents aged $<20$ years who had been diagnosed with IBD at the Kyungpook National University Hospital and Samsung Medical Center since 2000. The diagnosis of IBD was based on a combination of clinical evaluations including history, physical and endoscopic examination, histopathological findings, imaging, and serological assays for antineutrophil cytoplasmic autoantibody (ANCA) and anti-Saccharomyces cerevisiae antibody. ${ }^{3-7)}$

Additionally, we analyzed age, sex, medication use, IBD disease activity, and renal manifestations, such as hematuria, proteinuria, acute kidney injury, chronic kidney disease $\left(<90 \mathrm{~mL} / \mathrm{min} / 1.73 \mathrm{~m}^{2}\right.$ over 3 months), and reviewed other renal and urinary tract abnormalities. In particular, patients with proteinuria were screened in the first urine in the morning to exclude orthostatic proteinuria. The results of histopathological examination in patients who underwent renal biopsy were compared with the IBD disease activity. IBD disease activity can be assessed using the Pediatric Crohn Disease Activity Index (PCDAI) and the Pediatric Ulcerative Disease Activity Index (PUCAI) scores. These are noninvasive, valid, highly reliable, and responsive indices to assess disease activity in pediatric patients with CD and UC. PCDAI scores range from 0-100, and this index classifies disease activity as no disease activity $(<10)$, mild disease activity (11-30), and moderate-to-severe disease activity $(\geq 30)$. PUCAI scores range from $0-85$ and this index classifies disease activity as no disease activity $(<10)$, mild disease activity (11-34), moderate disease activity (35-64), and severe disease activity $(\geq 65)^{1,8)}$ The Institutional Review Board at Kyungpook National University Hospital, Daegu, South Korea reviewed and approved this study (approval number: KNUMC_2017-10-007).

Continuous variables were expressed as means \pm standard deviations (SD). The Student $t$ test and the Mann-Whitney test were used to analyze continuous variables. The chi-square test was used to analyze categorical variables. A $P$ value of $<0.05$ was considered statistically significant. All statistical analysis was performed using the R software, ver. 3.4.3 (R Foundation for Statistical Computing, Vienna, Austria).

\section{Results}

Our study comprised 456 children with IBD including 299 boys (65.6\%) and 157 girls (34.4\%) with a mean age \pm SD of $14.1 \pm 3.0$ years.
The mean follow-up period was $4.61 \pm 2.93$ years. The study included 346 children with CD (75.9\%) and 110 with UC (24.1\%), and the incidence of CD was greater than UC. Children with IBD were treated with various immunosuppressants including oral prednisolone, 5-aminosalicylic acid (5-ASA, sulfasalazine and mesalazine), azathioprine, infliximab, and adalimumab. ${ }^{3)}$ The renal function was assessed by measuring the estimated glomerular filtration rate (eGFR) less than $90 \mathrm{~mL} / \mathrm{min} / 1.73 \mathrm{~m}^{2}$ for more than 3 months. Of the 456 patients, 11 patients $(0.02 \%)$ had eGFR $<90$.

Among the 456 children, 67 (14.7\%) showed confirmed renal manifestations, and 59 were identified as children with $\mathrm{CD}$ and 8 as children with UC. We observed 26 children (38.8\%) with isolated hematuria, 30 (44.8\%) with isolated proteinuria, and 11 (16.4\%) with hematuria and concomitant proteinuria. During the course of evaluation to detect the cause of hematuria and concomitant proteinuria, we identified 1 child with a urinary stone and 1 with hydronephrosis.

A renal biopsy was performed in 7 children with CD. Of them, 5 children showed both microscopic hematuria and proteinuria. Persistent microscopic hematuria and recurrent gross hematuria was observed in 2 children. Complement 3/4 level, spot urine calcium/

Table 1. Patient characteristics $(n=456)$

\begin{tabular}{lc}
\hline Characteristic & Value \\
\hline No. of patients & 456 \\
CD & $346(75.9)$ \\
UC & $110(24.1)$ \\
Sex, male/female & $299(65.6) / 157(34.4)$ \\
Age (yr) & $14.1 \pm 3.0$ \\
FU duration (yr) & $4.6 \pm 2.9$ \\
Renal manifestations & $67(14.7)$ \\
CD & $59(88.1)$ \\
UC & $8(11.9)$ \\
Type of renal manifestations & \\
Hematuria & $26(39.8)$ \\
Proteinuria & $30(44.8)$ \\
Hematuria+proteinuria & $11(16.4)$ \\
Performed renal biopsy & \\
Total & 7 \\
Type of renal manifestations & \\
Microscopic hematuria and proteinuria & \\
Persistent microscopic hematuria and recurrent & $2(28.6)$ \\
gross hematuria & \\
Pathologic findings & \\
IgA nephropathy & \\
HSP nephritis & $5(71.4)$ \\
No abnormality & $1(14.2)$ \\
\hline Vales are presented as number (\%) or mean & $1(14.2)$ \\
\hline
\end{tabular}

Values are presented as number (\%) or mean \pm standard deviation. CD, Crohn disease; UC, ulcerative colitis; FU, follow-up; IgA, immunoglobulin A; HSP, Henoch-Schönlein purpura. 
creatinine level, ANCA, antinuclear antibody (ANA), and anti-ds DNA were examined in 7 patients who underwent renal biopsy. All of the patients were negative except 1 patient. This patient tested positive for ANCA, ANA, and anti-dsDNA, and IgA nephropathy (Haas classification I) was identified on renal biopsy. Histopathological examination revealed 5 children with IgA nephropathy (71.4\%) and in 1 child with Henoch-Schönlein purpura (HSP) nephritis (14.2\%), whereas 1 child (14.2\%) showed no histopathological abnormalities (Table 1). Based on the Haas classification of IgA nephropathy, 2 children were diagnosed with Haas subclass I, 2 with Haas subclass III, and 1 with Haas subclass IV (Fig. 1). Additionally, 1 child with HSP nephritis was diagnosed with class II disease based on the histological classification proposed by the International Study of Kidney Disease in Children. Angiotensin receptor blockers and/ or angiotensin converting enzyme inhibitors were administered to 5 children with IgA nephropathy and 1 with HSP nephritis, and oral prednisolone was administered to 2 of 6 children.

Disease activity was evaluated in 102 children, of which 81 showed CD and 21 showed UC. Of note, 35 of these 102 children (30 with $\mathrm{CD}, 5$ with UC) presented with renal manifestations. Disease activity was assessed based on the PCDAI for CD and the PUCAI for UC. The mean PCDAI score was 8.5 with renal manifestations and 2.2 without renal manifestations, and the mean PUCAI score was 21 with renal manifestations and 6.3 without renal manifestations. No significant correlation was observed between the IBD disease activity and the presence of renal manifestations in 102 children in whom we were able to assess disease activity. Disease activity was mild, and the disease was well controlled in all children (Table 2).

\section{Discussion}

IBD is a chronic relapsing disorder of unknown etiology that includes 2 distinct disorders- $\mathrm{CD}$ and UC. Approximately 2 decades ago, IBD was considered a rare disease among Korean adults. How- ever, the incidence of IBD has shown a rapid rise after the 1990s. Recently, the incidence of pediatric IBD has shown a steep rise in Korea since 2000. ${ }^{1)}$ Pediatric IBD usually demonstrates a more severe presentation of IBD than that in adults with widespread infiltration and more common colonic lesions.

Extraintestinal manifestations vary from 25\%-40\% in both adults and children with IBD. ${ }^{7,9,10)}$ In pediatric IBD patients, 6\% show at least one extraintestinal manifestation before a confirmed diagnosis of IBD is established, and an extraintestinal manifestation is known to develop in 29\% children within 15 years of diagnosis. ${ }^{11}$ The eyes (uveitis, episcleritis, retinitis), the skin (erythema nodosum, pyoderma gangrenosum), joints (arthritis, sacroiliitis, ankylosing spondylitis), hepatobiliary tract (sclerosing cholangitis, autoimmune chronic hepatitis), and respiratory tract (chronic bronchitis, bronchiectasis, bronchiolitis obliterans with organizing pneumonia) are most commonly involved in children with extraintestinal manifestations. ${ }^{12-15)}$

Among the IBD patients, renal manifestations account for 4\%$23 \%$ in adults, ${ }^{2)}$ but only in 1\% to $2 \%$ of children with IBD had renal manifestations such as proteinuria, hematuria, renal failure and uremia. ${ }^{9,12,14)}$ It was reported that renal manifestations have a 3-fold increased risk in males and a 10-fold increased risk in CD compared with UC. ${ }^{913)}$ Renal complications in IBD include nephrolithiasis,

Table 2. Comparison between the presence of renal manifestations and IBD disease activity

\begin{tabular}{lcccc}
\hline Variable & \multicolumn{2}{c}{ CD } & \multicolumn{2}{c}{ UC } \\
\hline Renal manifestation & $(+)$ & $(-)$ & $(+)$ & $(-)$ \\
Total & 30 & 51 & 5 & 16
\end{tabular}

$\begin{array}{llll}\text { IBD disease activity, mean } \pm S D^{*} \quad 8.5 \pm 21.2 & 2.2 \pm 7.0 & 21.0 \pm 28.8 & 6.3 \pm 18.4\end{array}$ $P$ value $\quad 0.25$ 0.38

$\mathrm{IBD}$, inflammatory bowel disease; CD, Crohn disease; UC, ulcerative colitis; SD, standard devitation.

* $\mathrm{BD}$ disease activity was assessed using the Pediatric Crohn Disease Activity Index and the Pediatric Ulcerative Disease Activity Index scores and evaluated in 102 of 456 enrolled children.
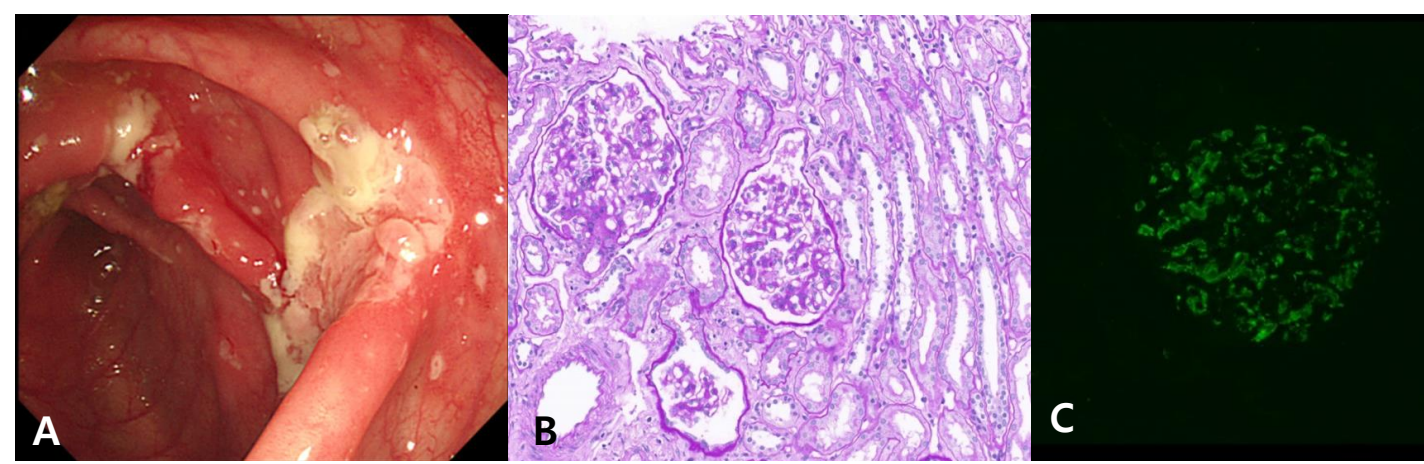

Fig. 1. Colonoscopic $(A)$ and renal pathologic $(B, C)$ findings in a patient with Crohn disease concomitant with IgA nephropathy. (A) lleocolonoscopy revealed multiple ulcers of various sizes throughout the colon, and large deep ulcers, in the cecum and ascending colon. (B) Increased mesangial matrix in the glomerulus on Periodic acid-Schiff stain $(\times 200)$. (C) Global granular staining for IgA in the mesangium on immunofluorescence $(\times 400)$. 
GN, interstitial nephritis, AA-type amyloidosis, and enterovesical fistula, among other such conditions. ${ }^{10,16)}$ In children, GN, renal artery stenosis, amyloidosis, renal failure, ureteral compression, and perinephric abscesses have been reported. ${ }^{7,14)}$

To date, the most common histopathological finding in the kidneys in these patients is interstitial nephritis secondary to the use of 5-ASA. 5-ASA and its derivatives (sulfasalazine and mesalazine) are widely used as first-line drugs of induction and maintenance in patients with IBD. ${ }^{8,17,18)}$ However, lately, following the popularity of new biological agents such as infliximab and adalimumab, the use of 5-ASA and its derivatives in patients with IBD and the consequent prevalence of renal complications caused by these drugs have been decreasing. In our study, no child was diagnosed with interstitial nephritis caused by 5-ASA.

A study evaluating the properties of renal stones observed in patients with $\mathrm{CD}$ demonstrated that the stones were predominantly composed of calcium oxalate and phosphate. The incidence of renal stones in patients undergoing an ileostomy is significantly high. The probability of renal stone formation was observed to be 8 fold higher in children with a urinary $\mathrm{pH} \leq 6.0$ than in those with a urinary $\mathrm{pH} \geq 6.5 .{ }^{19)}$ In our study, we incidentally identified 1 child with a urinary stone in the course of assessing the causes of hematuria and concomitant proteinuria.

Ambruzs et al. ${ }^{2)}$ reported the histopathological spectrum of renal biopsies in patients with IBD. Most patients with IBD with renal complications showed involvement of glomerular and tubulointerstitial components. IgA nephropathy was the most common diagnosis (24\%), followed by interstitial nephritis (19\%), arterionephrosclerosis (12\%), acute tubular injury (8\%), proliferative GN (7\%), and MCD (5\%). In addition, there have been several case reports suggesting the pathogenetic association between IgA nephropathy and $\mathrm{CD}$ in children. ${ }^{20,21)}$ Takemura et al. $^{20)}$ reported 10-year-old boy patient who had proteinuria with macroscopic hematuria and from the renal biopsy he was proven IgA nephropathy. At the age of 13 years, the patient developed CD and IgA nephropathy recurred, simutaneously. McCallum et al. ${ }^{21)}$ reported 11-year-old girl who presented with recurrent gross hematuria, she was proven IgA nephropathy from renal biopsy and later, she developed CD.

Shaer et al. ${ }^{16)}$ suggested that immune complex deposition may play an important role in the pathogenesis of GN associated with IBD. They concluded that all renal manifestations occurred in the setting of active bowel disease and that the improvement in GN was paralleled by the institution of treatment and subsequent improvement in the gastrointestinal disorder, confirming that GN is indeed an extra-intestinal manifestation of IBD. Forshaw et al. ${ }^{22)}$ also suggested that GN appeared to show a direct correlation with intestinal disease activity and improvement of renal function after remission of bowel inflammation has been conclusively demonstrated. The prevalence of IgA nephropathy was significantly higher in those with IBD than in those without IBD. ${ }^{10)}$ However, interestingly, because our study showed no correlation between renal manifestations and IBD disease activity, we conclude that this result suggests the role of other contributory pathogenetic mechanisms in addition to IBD disease activity. Therefore, it is necessary to confirm renal manifestations by performing regular urinalysis even in patients with IBD without disease activity.

This study was limited in that periodic urinalysis was not performed in all children with IBD. It was unclear when the renal manifestations occurred, and we cannot exclude the possibility of pre-existing renal manifestations that might have progressed and been noticed only during the study period. Thus, it is possible that the relevant tests were not performed despite an indication for a biopsy, and thus the observed biopsy result may not be representative of the whole.

However, this study is the first to report the prevalence and clinical manifestations of kidney-related symptoms in children with IBD using a large-scale Korean pediatric IBD series, and further larger prospective observational studies are needed in the future. Children with IBD are more likely to show renal symptoms than healthy children and adolescents. Therefore, regular urinary screening and evaluation of renal function are necessary for early detection and prompt treatment of renal complications.

\section{Conflicts of interest}

No potential conflict of interest relevant to this article was reported.

\section{References}

1. Oh SH, Kim KM. Current issues of pediatric inflammatory bowel disease in Korea. Korean J Pediatr 2014;57:465-71.

2. Ambruzs JM, Walker PD, Larsen CP. The histopathologic spectrum of kidney biopsies in patients with inflammatory bowel disease. Clin J Am Soc Nephrol 2014;9:265-70.

3. Kim ES, Kim WH. Inflammatory bowel disease in Korea: epidemiological, genomic, clinical, and therapeutic characteristics. Gut Liver 2010;4:1-14.

4. Ye BD, Jang BI, Jeen YT, Lee KM, Kim JS, Yang SK, et al. Diagnostic guideline of Crohn's disease. Korean J Gastroenterol 2009;53:161-76.

5. Dignass A, Eliakim R, Magro F, Maaser C, Chowers Y, Geboes K, et al. Second European evidence-based consensus on the diagnosis and management of ulcerative colitis part 1: definitions and diagnosis. $J$ Crohns Colitis 2012;6:965-90.

6. Park SM, Han DS, Yang SK, Hong WS, Min YI. Clinical features of ulcerative colitis in Korea. Korean J Intern Med 1996;11:9-17.

7. Diefenbach KA, Breuer CK. Pediatric inflammatory bowel disease. World J Gastroenterol 2006;12:3204-12.

8. Arend LJ, Springate JE. Interstitial nephritis from mesalazine: case report and literature review. Pediatr Nephrol 2004;19:550-3.

9. Levine JS, Burakoff R. Extraintestinal manifestations of inflammatory bowel disease. Gastroenterol Hepatol (N Y) 2011;7:235-41.

10. Corica D, Romano C. Renal involvement in inflammatory bowel 
diseases. J Crohns Colitis 2016;10:226-35.

11. Dotson JL, Hyams JS, Markowitz J, LeLeiko NS, Mack DR, Evans JS, et al. Extraintestinal manifestations of pediatric inflammatory bowel disease and their relation to disease type and severity. J Pediatr Gastroenterol Nutr 2010;51:140-5.

12. Jose FA, Heyman MB. Extraintestinal manifestations of inflammatory bowel disease. J Pediatr Gastroenterol Nutr 2008;46:124-33.

13. Danese S, Semeraro S, Papa A, Roberto I, Scaldaferri F, Fedeli G, et al. Extraintestinal manifestations in inflammatory bowel disease. World J Gastroenterol 2005;11:7227-36.

14. Aloi M, Cucchiara S. Extradigestive manifestations of IBD in pediatrics. Eur Rev Med Pharmacol Sci 2009;13 Suppl 1:23-32.

15. Jose FA, Garnett EA, Vittinghoff E, Ferry GD, Winter HS, Baldassano $\mathrm{RN}$, et al. Development of extraintestinal manifestations in pediatric patients with inflammatory bowel disease. Inflamm Bowel Dis 2009; 15:63-8.

16. Shaer AJ, Stewart LR, Cheek DE, Hurray D, Self SE. IgA antiglomerular basement membrane nephritis associated with Crohn's disease: a case report and review of glomerulonephritis in inflammatory bowel dis- ease. Am J Kidney Dis 2003;41:1097-109.

17. Izzedine H, Simon J, Piette AM, Lucsko M, Baumelou A, Charitanski D, et al. Primary chronic interstitial nephritis in Crohn's disease. Gastroenterology 2002;123:1436-40.

18. Uslu N, Demir H, Saltik-Temizel IN, Topaloğlu R, Gürakan F, Yüce A. Acute tubular injury associated with mesalazine therapy in an adolescent girl with inflammatory bowel disease. Dig Dis Sci 2007;52: 2926-9.

19. Ishii G, Nakajima K, Tanaka N, Hara H, Kato M, Ishii N. Clinical evaluation of urolithiasis in Crohn's disease. Int J Urol 2009;16:477-80.

20. Takemura T, Okada M, Yagi K, Kuwajima H, Yanagida H. An adolescent with IgA nephropathy and Crohn disease: pathogenetic implications. Pediatr Nephrol 2002;17:863-6.

21. McCallum D, Smith L, Harley F, Yiu V. IgA nephropathy and thin basement membrane disease in association with Crohn disease. Pediatr Nephrol 1997;11:637-40.

22. Forshaw MJ, Guirguis 0, Hennigan TW. IgA nephropathy in association with Crohn's disease. Int J Colorectal Dis 2005;20:463-5. 